\title{
Matriz de posicionamento estratégico de materiais: conceito, método e estudo de caso
}

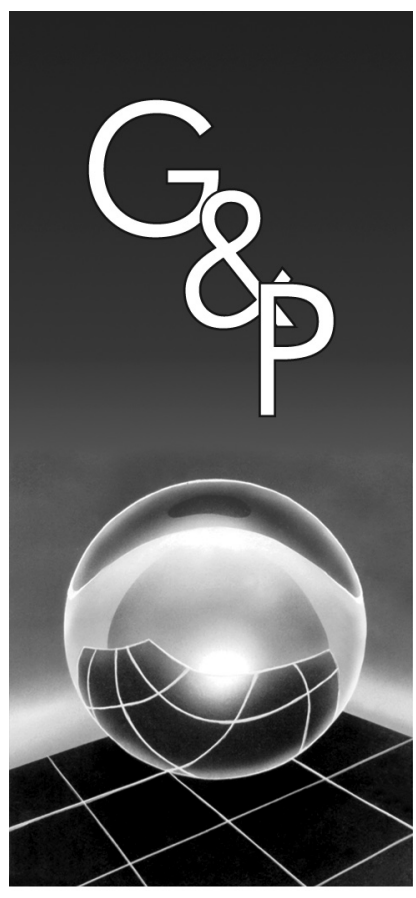

Marcelo Klippel

José Antonio Valle Antunes Júnior

Guilherme Luís Roehe Vaccaro

\section{Resumo}

A gestão de materiais representa uma questão de interesse na gestão de operações, uma vez que pode reduzir ou promover a flexibilidade do ambiente produtivo, e por conseqüência, impactar os resultados globais da organização. Este artigo apresenta os principais conceitos para sustentação e implantação da matriz de posicionamento estratégico de materiais (MPEM) e discute a necessidade de gerenciar eficazmente os materiais no contexto produtivo. Adicionalmente, apresenta um estudo de caso de uma empresa industrial do ramo metal mecânico sugerindo elementos metodológicos para implantação da MPEM. Finalmente, é salientada a importância da gestão contínua dos materiais, envolvendo a segmentação da gestão nos diferentes quadrantes da matriz e utilizando um mecanismo de indicadores de desempenho adotadas pela empresa.

Palavras-chave: Gestão de materiais. Gestão estratégica. Matriz de posicionamento estratégico.

\section{Introdução}

Durante as duas últimas décadas do Século $\mathrm{XX}$, as empresas industriais passaram por profundas mudanças, ainda sob efeito da competição intercapitalista ocorrida a partir das crises internacionais do petróleo, em 1973 e 1979. Um dos principais efeitos foi a busca intensiva por melhorias de desempenho competitivo nos mercados. No Brasil, estas condições competitivas foram enfrentadas basicamente através da adoção dos modernos princípios de engenharia de produção, tais como: Sistema Toyota de Produção, produção enxuta, just-in-time, controle da qualidade total, teoria das restrições, etc. Porém, o eixo dos trabalhos realizados esteve fortemente focado nas melhorias internas dos sistemas produtivos. Embora esses esforços continuem relevantes e necessários, tornase cada vez mais clara a necessidade de ampliação dos trabalhos de melhorias contínuas para a cadeia de fornecedores como um todo.

Uma parte significativa dos custos industriais associados aos produtos fabricados está relacionada com o fornecimento de materiais. Nas empresas industriais, em média esta parcela gira em torno de $60 \%$ do custo dos produtos fabricados (HARMON, 1993). Considerado este fato, justifica-se a tendência de investimento das empresas em projetos de desenvolvimento de sua cadeia de fornecedores, especificamente em termos de gestão do fornecimento dos materiais (DOBLER, 1996).

Através de um exercício teórico com a finalidade de avaliar o impacto do custo dos materiais e do montante de estoque envolvidos em uma operação típica de empresas industriais, Dobler (1996) evidencia que a empresa pode aumentar seu retorno sobre investimento (RSI) de três maneiras básicas:

a) redução dos custos associados às atividades de vendas;

b) aumento das vendas com os ativos disponíveis (ou ainda, o aumento das vendas proporcionalmente mais rápido do que os investimentos); e

c) estratégia combinada de redução de custos e aumento das vendas com os mesmos ativos.

O exercício proposto por Dobler (1996) apresenta o impacto da redução dos custos de compra de materiais e dos inventários (estoque em processo e produtos acabados) no RSI de Empresas Industriais, através de uma simulação de redução de 5\% nos custos de materiais, ou seja, nos custos envolvidos com a operação, ao mesmo tempo em que reduz também 5\% nos inventários. O resultado é observado através do aumento no RSI na 
ordem de $10 \%$ para $13 \%$. No exercício, a relação entre os custos de materiais e o lucro da empresa é direta. Por outro lado, para aumentar o lucro nos mesmos valores atingidos com a redução dos custos de materiais através de novas vendas, seria necessário um aumento equivalente a $28,8 \%$ nas quantidades vendidas. Em grande parte, esta diferença se dá graças ao fato de que ao aumentar o volume de vendas, aumentam-se também os custos dos materiais envolvidos, pois um maior volume de insumos também será necessário. Evidentemente, a situação ideal consiste na minimização dos custos dos materiais conjuntamente com o aumento no volume de vendas.

Aponta-se, à gestão de materiais, dois sentidos de atuação:

a) redução dos preços de custos dos materiais e matérias-primas, através de ações em parceria com fornecedores ou de inovações sobre os produtos; e

b) redução dos níveis gerais de inventários de matériaprima, de estoque em processo e de produto acabado, através de melhorias na cadeia logística e no Planejamento, Programação e Controle da Produção.

Este artigo tem por objetivo apresentar uma forma de gestão estratégica dos materiais produtivos que é capaz de endereçar estrategicamente as questões acima mencionadas, através do uso de uma matriz de posicionamento estratégico para os materiais no contexto produtivo. Os objetivos específicos são: i) apresentar os principais conceitos que sustentam a matriz de posicionamento estratégico de materiais (MPEM); ii) propor um método de implantação da MPEM; iii) apresentar um caso de aplicação prática do método; e iv) realizar uma análise crítica dos diferenciais competitivos de utilização deste método.

\section{Referencial teórico}

Nas seções seguintes, são apresentados os elementos conceituais necessários ou correlatos ao método proposto. Serão tratados os temas: i) método $\mathrm{ABC}$ para gestão dos materiais; ii) método $\mathrm{ABC}$ com criticidade; iii) matriz de Kraljic; e iv) matriz de posicionamento estratégico de materiais (MPEM).

\subsection{Método ABC para gestão de materiais}

Um número significativo de empresas industriais nacionais e internacionais tem tratado a questão das compras envolvendo matérias-primas, componentes e serviços, de uma forma padronizada e única. Em outras palavras, a despeito da disponibilidade de ferramentas na base de conhecimentos da engenharia da produção, ainda é comum observar o tratamento do tópico gestão de compras de uma forma homogênea e não segmentada.

Uma ferramenta freqüentemente encontrada em ambientes empresariais é a elaboração das curvas $\mathrm{ABC}$ de materiais, que tem sua origem conceitual relacionada às teorias propostas no século XIX, pelo economista e sociólogo italiano Vilfredo Pareto. As curvas ABC representam uma primeira ferramenta interessante de análise, que permite iniciar o processo de priorização da gestão dos materiais e serviços adquiridos por uma dada organização.

A análise e classificação $\mathrm{ABC}$ é um conceito simples, podendo ser muito útil no gerenciamento de estoques, principalmente no que tange à concentração dos esforços nos itens mais importantes e caros (SALVENDY, 1992). Cavinato e Kauffman (1999) exemplificam o uso da análise $\mathrm{ABC}$ para o processo de aquisição de materiais indicando que normalmente a análise $\mathrm{ABC}$ exerce influência sobre os materiais comprados, número de fornecedores, estoques e outras medidas. Freqüentemente, a análise $\mathrm{ABC}$ se refere ao princípio 80-20 de Pareto, no qual aproximadamente $20 \%$ dos itens/serviços comprados representam cerca de $80 \%$ do valor financeiro gasto.

Salvendy (1992) salienta que o principal benefício da análise $\mathrm{ABC}$ consiste no fato de permitir uma gestão eficaz no que tange à identificação dos itens/materiais que merecem maior atenção, chamados de itens do tipo A. $\mathrm{O}$ rigor na estimativa dos custos associados ao estoque, assim como outros parâmetros que determinam os níveis ótimos de estoque, torna-se prioritário para os itens do tipo A, em relação aos itens do tipo $\mathrm{C}$, uma vez que estes últimos geralmente exigem muito esforço de gestão com resultados pouco expressivos para os resultados econômico-financeiros da Organização. Cavinato e Kauffman (1999) afirmam ainda, que a utilização da análise ABC é importante para a redução do volume e complexidade das transações individuais, uma vez que transações desnecessárias podem ser desconsideradas.

A classificação $\mathrm{ABC}$, embora relevante na medida em que focaliza seus esforços em compreender a estrutura de custos de compras dos itens e dos serviços das empresas, apresenta limitações, quando se observa a gestão das compras de forma ampla. Entre estas limitações pode-se citar:

a) não considera os aspectos relativos à qualidade das compras realizadas;

b) não considera a relevância estratégica dos itens e serviços adquiridos; e

c) não considera os aspectos relativos ao valor gerado pelas aquisições no sentido da agregação de valor aos produtos e serviços.

Com o objetivo de fornecer uma orientação adicional para os gerentes de suprimentos, Dobler e Burt (1996) sugerem que cada material pode ainda ser classificado de acordo com sua importância operacional conforme a seguinte escala: 1: crítica; 2: média; e 3: não-crítica. Desta forma, um material A menos importante poderia ser tratado como sendo um item A-3, enquanto um mate- 
rial C crítico poderia ser identificado como sendo um item C-1. Esta classificação com dois dígitos permite que em alguns casos um item C-1 necessite de mais atenção gerencial do que um item do tipo A-3. Esta variação da análise $\mathrm{ABC}$ tradicional é conhecida como Método $\mathrm{ABC}$ com Criticidade.

\subsection{Método ABC com criticidade}

Segundo Martins e Alt (2004), a análise ABC tradicional pode trazer distorções perigosas para a empresa, uma vez que não considera a importância do item em relação à operação do sistema como um todo. Em outros termos, a criticidade do item não é levada em consideração.

O Método $\mathrm{ABC}$ com Criticidade é uma variante que objetiva solucionar a deficiência do método tradicional através da análise de criticidade do item, ou seja, da avaliação do impacto de sua falta na operação do sistema. Martins e Alt (2004) deixam claro que a falta de um item crítico pode influenciar negativamente na imagem da empresa para o cliente, na facilidade de substituição deste item por outro e na velocidade de obsolescência. Por exemplo, um parafuso de baixo custo e consumo, normalmente é considerado um item do tipo C. Porém, eventualmente este item pode interromper a operação de um equipamento ou instalação essencial à produção dos bens e serviços.

Em termos de criticidade, os itens podem ser classificados em: 1 (ou A): itens cuja falta provoca a interrupção da produção, cuja substituição é difícil ou não existem fornecedores alternativos; 2 (ou B): itens cuja falta não exerce efeito na produção a curto prazo; e 3 (ou C): demais itens. Em termos do nível de criticidade dos itens, os fatores a serem considerados devem ser definidos pela própria empresa. Por exemplo: prazo de atendimento, prazo de pagamento, pós-venda, qualidade intrínseca do produto, logística e inovação. Desta forma, a criticidade dos itens tende a levar em consideração as características específicas de cada empresa, através da adoção dos fatores que mais são influenciados na falta do item.

Após a classificação, é realizada uma renormalização dos grupos, da seguinte forma (MARTINS; ALT, 2004):

a) classe AA: itens A-1, A-2 e B-1;

b) classe $\mathrm{BB}$ : itens A-3, B-2 e C-1; e

c) classe CC: itens B-3, C-2 e C-3.

Embora a abordagem $\mathrm{ABC}$ com criticidade possua avanços significativos em relação à abordagem $\mathrm{ABC}$ tradicional, abordagens mais estruturadas, abrangentes e conceitualmente consistentes podem ser construídas. Estas abordagens foram construídas a partir da Matriz de Kraljic, discutida a seguir.

\subsection{A matriz de Kraljic}

O modelo de compras de Kraljic (1983) apud Have et al. (2003) e suas variações visam dar suporte à seleção estratégica de compras, diferenciando produtos por tipos distintos na organização. O objetivo final da matriz consiste em otimizar a relação entre custos (diretos e indiretos) e risco. A matriz cruza duas dimensões: impacto sobre o resultado financeiro e incerteza de oferta, gerando quatro quadrantes para a categorização de produtos, conforme apresentado na Figura 1.

A construção da matriz de Kraljic possui diversos elementos de subjetividade, iniciando pelo agrupamento lógico dos produtos e por sua classificação em termos de risco financeiro e incerteza sobre a oferta. Sugestões para estas atividades partem da constituição de listas de fornecedores, análises qualitativas sobre o esforço de migração das compras de um item de um fornecedor

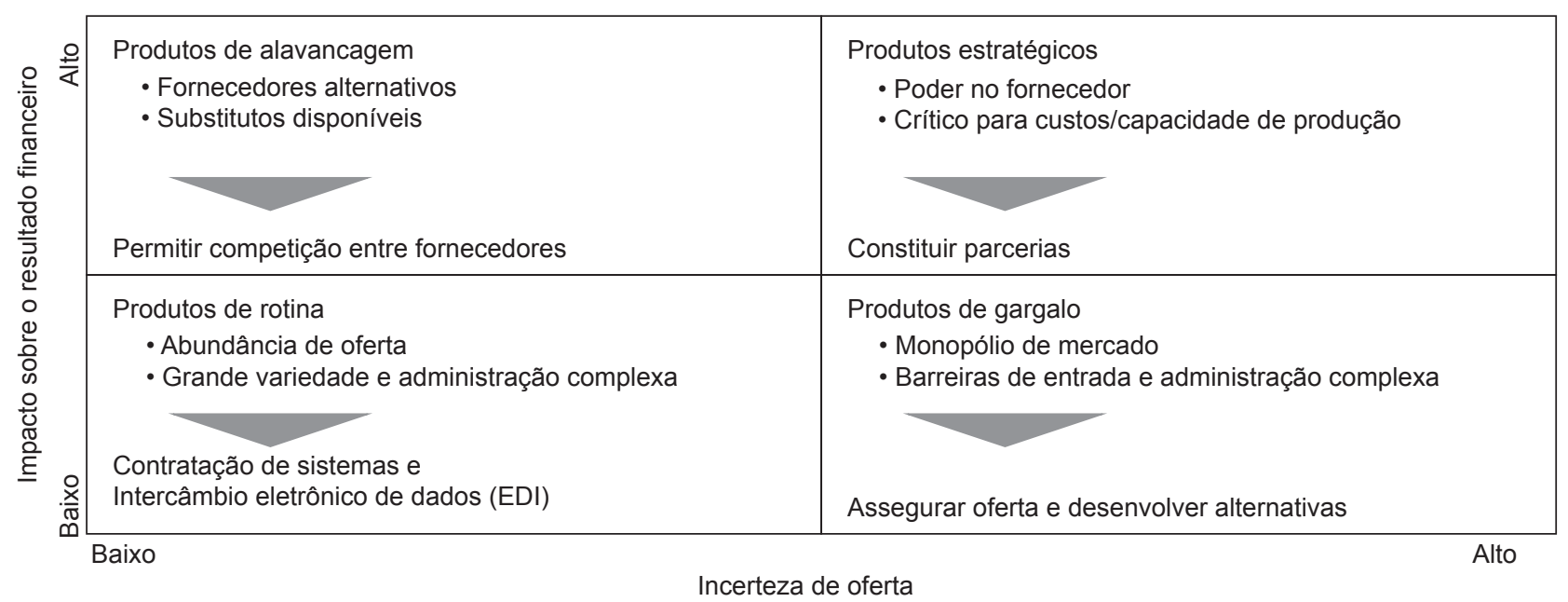

Figura 1. Matriz de Kraljic (Adaptado de HAVE et al., 2003). 
para outro, custos diretos de aquisição, custos indiretos de aquisição, análise de reservas alternativas e estabilidade do fornecedor (HAVE et al., 2003). As marcas de separação dos quadrantes são arbitrária, podendo variar conforme a realidade de cada organização.

Este modelo permite o foco na administração de compras da organização, evitando a "economia de palitos" (HAVE et al., 2003) e dá margem para que se obtenham vantagens estratégicas pela gestão de fornecedores.

\subsection{A matriz de posicionamento estratégico de materiais}

A MPEM, um aprimoramento elaborado a partir da matriz de Kraljic, é um instrumento para a gestão segmentada de suprimentos no contexto produtivo, apresentando duas dimensões fundamentais. Sua construção está baseada em dois referenciais teóricos básicos, a saber:

a) a noção de forças competitivas proposta por Porter (1986); e

b) as chamadas dimensões da estratégia de produção.

A lógica proposta por Porter (1986) baseia-se nas chamadas cinco forças competitivas que, segundo o autor, determinam a rentabilidade da empresa: entrada de novos produtos; ameaça de substitutos; poder de negociação de fornecedores; poder de negociação dos compradores; e rivalidade entre os concorrentes existentes.

No eixo horizontal da matriz é considerada a dimensão global risco (ou exposição) dos materiais (CARTER, 1999; GRIECO, 1995). Segundo Grieco (1995), para a análise dos riscos relacionados ao fornecimento de materiais, os seguintes elementos devem ser levados em consideração:

a) poder de barganha dos fornecedores;

b) potencial de substituição do fornecedor dos materiais;

c) rivalidade no fornecimento dos materiais; e

d) barreiras à entrada de fornecedores.

Ou seja, é adotada a noção das cinco forças competitivas propugnadas por Porter, tendo como objeto a área de materiais.

No eixo vertical da matriz, considera-se a dimensão global custo/valor dos materiais dentro do contexto dos produtos da empresa (CARTER, 1999).

No campo da estratégia de produção são usualmente consideradas seis dimensões competitivas: qualidade; custo; atendimento; flexibilidade; inovação; e tempo de atravessamento (lead time). Partindo de quatro dimensões da estratégia competitiva (qualidade, custo, tempo e tecnologia/inovação), Grieco (1995) e Carter (1999) constroem a dimensão global custo/valor dos materiais, a qual permite identificar a influência nos resultados, considerado o contexto dos produtos da empresa. A dimensão tempo tende a englobar as noções de atendimento e tempo de atravessamento. A dimensão flexibilidade não é diretamente considerada na análise. As dimensões competitivas a serem tratadas podem ser ampliadas para a realidade de cada empresa em questão.

A partir destes dois eixos (Risco de suprimento e Influência sobre os resultados) a MPEM classifica os materiais em quatro grandes segmentos, conforme apresentado na Figura 2:

a) componentes não-críticos: com baixo risco de fornecimento e baixa influência nos resultados da empresa;

b) componentes estratégicos: com elevado risco de fornecimento e elevada influência nos resultados da empresa;

c) componentes de risco: com elevado risco de fornecimento e baixa influência nos resultados na empresa; $\mathrm{e}$

d) componentes competitivos: com baixo risco de fornecimento e alta influência nos resultados da empresa.

É importante ressaltar que o conjunto de decisões da MPEM contém subjetividades. Da mesma forma que na construção da matriz de Kraljic, as decisões sobre os impactos das dimensões de Risco e Influência são dependentes da percepção da organização (ou de seus profissionais, em última análise) sobre o contexto da gestão dos materiais no ambiente produtivo. Como forma de redução dos componentes subjetivos, o método proposto para a construção da MPEM prevê o uso de votações (utiliza-se um método de multivotação, englobando um amplo número de profissionais da empresa, envolvidos com o problema).

Outro ponto de subjetividade forte é a determinação do ponto de corte dos quadrantes da MPEM. Essa separação depende intrinsecamente da estratégia adotada pela empresa. Por exemplo, a consideração de um ponto de corte inferior à metade da escala de risco de suprimento posiciona a empresa de forma crítica a diversos materiais, exigindo maior esforço de seus colaboradores no sentido da melhoria da gestão dos materiais, visto que muitos

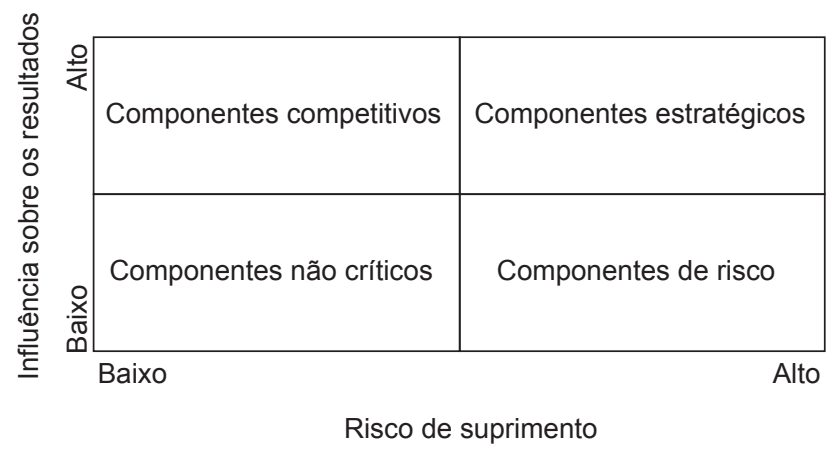

Figura 2. Matriz de posicionamento estratégico de materiais (MPEM). 
deles passarão a ser interpretados como estratégicos ou de risco. Por outro lado, o estabelecimento de um valor de corte superior à metade da escala de Risco de suprimento posiciona a empresa em uma situação mais "confortável", na qual um número menor de elementos necessita ser monitorado de forma mais crítica. Uma estratégia inicial é o uso de um corte simétrico. Com a evolução do uso do método, a empresa pode, por exemplo, migrar sistematicamente para um critério de corte fundamentado no princípio de Pareto, tendo $4 / 5$ de seus materiais como de baixo risco de suprimento.

Essa mesma discussão pode ser estabelecida para a dimensão de Influência sobre os Resultados. Valores inferiores à metade da escala podem posicionar a empresa de forma mais ativa na melhoria de parcerias ou no desenvolvimento de inovações sobre seus produtos, de modo a reduzir a necessidade por materiais de alta influência sobre os resultados. Valores superiores à metade da escala podem indicar uma posição menos suscetível a variações financeiras.

Considerando o aspecto da gestão de materiais fundamentada na MPEM, as seguintes questões são pertinentes: Quais os mecanismos de gestão que devem ser utilizados em cada quadrante da MPEM? Como tratar os materiais de forma estratégica, a partir dos quatro quadrantes da matriz?

Sugere-se a seguinte abordagem para os quadrantes da matriz, sujeita às realidades específicas de cada empresa:

a) componentes competitivos podem ser gerenciados pelos setores responsáveis e pela realização de melhorias de produtividade e qualidade, uma vez que as estratégias de redução de custos são essenciais para diminuir o impacto que estes componentes exercem sobre o resultado da organização;

b) componentes não-críticos devem ser organizados segundo uma lógica geral de redução da variedade de fornecedores e ganhos de escala, associados ao incremento do volume de compras de materiais. Usualmente, neste caso a gestão pode ser relacionada diretamente ao setor de compras corporativo da empresa;

c) componentes de risco são extremamente críticos, visto que podem atrasar a fabricação e entrega dos produtos aos clientes, uma vez que apresentam alto risco de suprimento. Desta maneira, sugere-se que todos os esforços sejam exercidos e gerenciados pela engenharia de projeto e de produto, já que conceitualmente estes componentes precisam ser repensados e, em muitos casos, substituídos por outros; e

d) componentes estratégicos são essenciais do ponto de vista do desempenho estratégico da empresa e devem ser tratados pela alta direção. A gestão destes materiais, tendo como base a ação da direção da empresa, explica-se na medida em que envolve ações e decisões estratégicas (por exemplo: construção de contratos a longo prazo com fornecedores que possuem alto poder de barganha em relação ao cliente).

A implantação da MPEM é o foco da próxima seção, na qual é sugerido um método para sua realização.

\section{0 método geral de trabalho preliminar (MGTP)}

Nesta seção, o método geral de trabalho preliminar (MGTP) é apresentado no contexto da implantação da MPEM. Este método foi originalmente apresentado por Antunes e Klippel (2002) e consiste de 13 etapas seqüenciais, visando operacionalizar projetos de implantação em ambientes industriais. A Figura 3 apresenta o fluxograma do MGTP adaptado para a implementação da MPEM. O MGTP ora apresentado foi constituído a partir da síntese realizada em termos teóricos e de um conjunto de proposições metodológicas autônomas geradas a partir de lacunas observadas na literatura aberta sobre $o$ tema. Este método foi testado em duas empresas do ramo metal-mecânico do estado do Rio Grande do Sul - uma do segmento automotivo e outra da área de transportes. A seguir, cada etapa será sucintamente comentada:

Etapa 1: Consiste na apresentação do método de trabalho para o grupo gestor da empresa, com a finalidade de explicitar os conceitos, princípios e resultados esperados, com a implementação do Método de Posicionamento Estratégico de Materiais. Nesta fase, procura-se elucidar a significância do tema (aspectos estratégicos, econômico-financeiros, de qualidade, etc.) e a necessidade de tratamento específico das questões relacionadas ao suprimento de materiais em cada quadrante da matriz.

Etapa 2: Estabelecido o alinhamento com o grupo gestor da empresa, deve ser formado o grupo de trabalho (GT) que conduzirá o projeto. As pessoas envolvidas devem definir e analisar os critérios como: a interfuncionalidade e o conhecimento detalhado dos produtos e dos materiais envolvidos. O GT deve englobar profissionais de áreas distintas da empresa: engenharia de produto e processo, qualidade, compras, programação, planejamento e controle da produção e materiais, tecnologia, custos e gerência da produção. é fundamental o apoio do grupo gestor, no sentido de manter as pessoas escolhidas no GT até a conclusão dos trabalhos, sob pena de comprometimento da qualidade e da seqüência de informações geradas.

Etapa 3: É iniciado o refinamento da abrangência do projeto, através do levantamento dos produtos e dos materiais a serem tratados. Em um primeiro momento, o GT deve selecionar os itens mais significativos para 


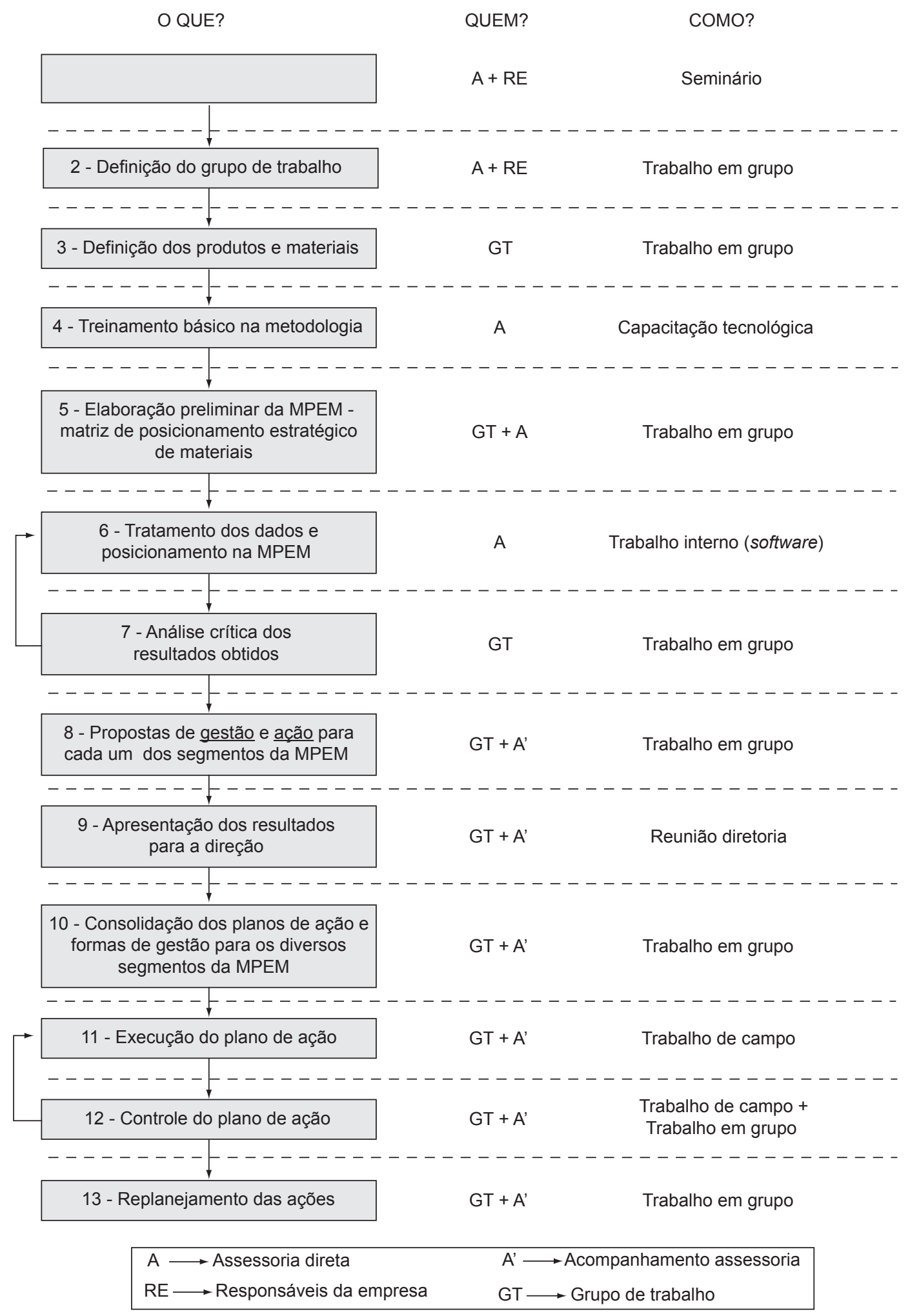

Figura 3. Fluxograma do MGTP adaptado para a implantação da MPEM.

a empresa, a partir de uma ótica econômica e/ou estratégica. Neste ponto, a consulta a formas já utilizadas de gestão dos materiais pela empresa é benéfica. Por exemplo, se a empresa já fizer uso do método $\mathrm{ABC}$ para a gestão dos materiais, pode-se partir de seus registros para identificar a abrangência dos itens a serem tratados. Além disso, esses dados de posicionamento poderão ser utilizados como entradas para a Etapa 5.

Etapa 4: Consiste no treinamento básico do método. Este treinamento deve englobar os conceitos, os princí- 
pios, o método proposto e a forma específica utilizada para tratamento específico dos dados. Propõe-se que este treinamento foque o repasse tecnológico para o GT.

Etapa 5: A fase inicial da aplicação propriamente dita da MPEM consiste em um conjunto de votações que visam posicionar e classificar posteriormente cada material, segundo alguns critérios.

Cada participante deve receber um formulário de coleta de dados da Influência nos Resultados, para cada produto específico. Este procedimento de votação é repetido para todos os produtos selecionados. Materiais relacionados em diferentes produtos devem ser tratados em separado, pois a influência no resultado poderia ser totalmente divergente.

Da mesma forma, outro formulário deve ser distribuído, contendo a relação de todos os materiais selecionados. Neste segundo formulário, os materiais são apresentados independentemente do produto ao qual estão associados, visando analisar o risco de suprimento do material em particular.

Cada integrante do GT deve realizar as votações de maneira individual, não ocorrendo interferência dos demais profissionais. Conforme relatado anteriormente, pode-se fazer uso de classificações prévias $(\mathrm{ABC}$, por exemplo) para fundamentar as votações. Nesse caso, a própria etapa servirá como uma avaliação crítica individual da classificação de materiais existente no contexto da empresa. O processo de votação é utilizado nesta etapa e novamente na Etapa 7 como mecanismo de minimização do componente subjetivo de classificação dos materiais.

Etapa 6: Os dados provenientes das votações são tabulados de modo a definir o índice que traduz a influência do produto/material no resultado da empresa. Este número é formado a partir de uma influência ponderada de dois pesos: o peso relativo do produto na competição e a influência do material. Para a votação do peso relativo do produto na competição, devem-se considerar apenas os produtos em relação ao mercado, seguindose quatro fatores pré-definidos: custo, qualidade, tempo e tecnologia. Neste caso, cada votação deve somar um total de 1,0, devendo-se distribuir os pesos de acordo com a importância que cada profissional do GT confere a cada fator, dentro do produto em análise. É possível, neste ponto, o uso de outras técnicas de suporte à decisão multicritério, tais como o analytical hierarchy process (AHP). Quanto à influência do material, devem-se considerar os mesmos fatores citados acima, porém os pesos devem ser de 0 a 5 ( 0 representa pouca influência e 5 muita influência), devendo-se considerar cada material específico em função da sua relevância em relação ao produto em pauta. Assim, um mesmo material pode ter uma influência diferente no resultado, dependendo do produto considerado (por exemplo, um mesmo material pode ser um item comum para um produto $\mathrm{X}$ e um item de segurança para o produto $\mathrm{Y}$ ). Neste caso, as votações serão distintas, embora o item em análise seja o mesmo. Com estes dois pesos, chega-se à influência ponderada, que representa um dos dois índices da matriz.

Em um segundo momento deve-se considerar o risco de suprimento de cada material. Neste caso, considera-se cada material de acordo com outros quatro fatores:

a) poder de barganha: diz respeito ao poder de negociação, que pode estar com o comprador ou com o fornecedor, tendo intensidade de 0 a 5 , respectivamente;

b) substituição: diz respeito à possibilidade de substituir o material em questão por algum outro tipo de material, sem que seja necessário alterar o processo original. Da mesma maneira, para este fator vota-se entre 0 e 5 , sendo 0 para fácil substituição e 5 para substituição muito difícil;

c) rivalidade: diz respeito à concorrência existente entre os fornecedores de cada material específico, ou seja, havendo muitos fornecedores para o mesmo material, o risco de suprimento é muito pequeno, representando valores mais próximos de 0 ; e

d) barreiras à entrada: representam a possibilidade de desenvolvimento de um novo fornecedor específico para o material em questão, devendo este ser fraco para facilmente penetrável, ou forte para difícil desenvolvimento. O grupo de trabalho deve votar, individualmente, todos os materiais com pesos de 0 a 5.

A ponderação deste índice é utilizada no eixo das abscissas. O resultado das votações e tabulações é uma lista dos materiais de cada produto, classificados em quatro quadrantes de acordo com a Figura 4. A definição dos pontos de corte que delimitam os quadrantes também deve ser alvo de votação e consenso do GT.

Etapa 7: Nesta etapa realiza-se uma análise crítica das matrizes geradas, de modo a validar as votações

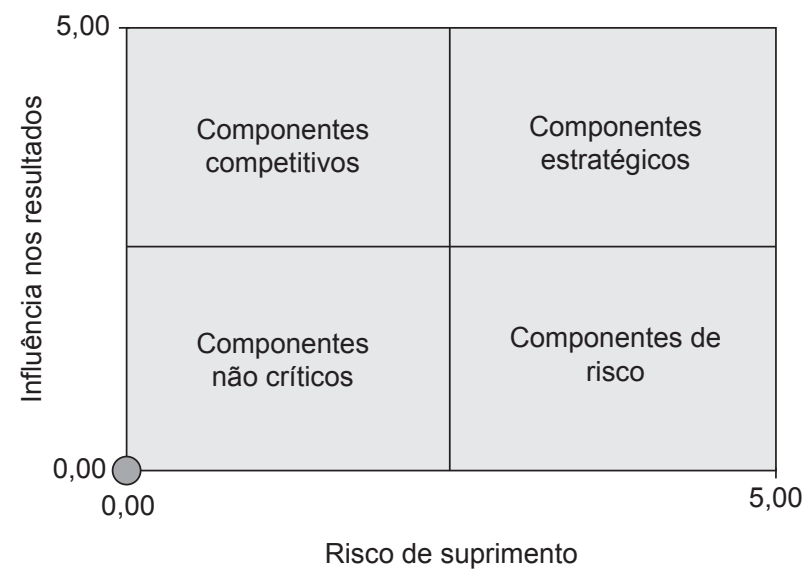

Figura 4. Matriz de posicionamento estratégico de materiais. 
realizadas pelos integrantes do GT. Esta etapa visa ainda identificar e dirimir discrepâncias entre os resultados obtidos e a realidade da empresa. Após a análise crítica dos resultados, os itens sobre os quais pesam dúvidas devem ser analisados detalhadamente. Pode-se rever a definição dos materiais, visando segmentar determinados itens em dois ou mais, caso isto facilite o posicionamento ou gere conhecimento para a organização (por exemplo, o material plástico genérico pode necessitar ser dividido em plástico para embalagem e plástico técnico). Uma vez realizada a segmentação, todos os itens duvidosos devem ser submetidos a um novo processo de votação. Após as eventuais votações, deve-se retornar à Etapa 6 para novo tratamento de dados e posicionamento do material.

Etapa 8: Com base nas informações geradas, são elaboradas propostas estratégicas para a gestão e ação de cada um dos segmentos da MPEM. Estas estratégias, para cada quadrante, devem ser segmentadas em planos de ações, os quais devem ser acompanhados de cronogramas para operacionalização. Para diferentes segmentos da matriz, podem ser requeridas diferentes formas de gestão.

Etapa 9: Consiste na apresentação dos resultados obtidos pelo GT para o grupo gestor da empresa. Nesta apresentação devem ser discutidas as formas de gestão para os quadrantes específicos (materiais estratégicos, competitivos, não-críticos e de risco), bem como a necessidade de maneiras diferenciadas de gestão. $\mathrm{O}$ encaminhamento dessas ações deve ficar sob responsabilidade do grupo gestor da empresa.

Etapa 10: Após aprovados os resultados pelo grupo gestor, os planos de ação e as formas de gestão devem ser consolidados, estabelecendo-se uma hierarquia de prioridades em relação às ações tomadas. Nesta etapa, deve-se construir um cronograma definitivo de implantação das ações, encerrando o ciclo de planejamento.

Etapa 11: Consiste na execução do plano de ações. Neste momento, o GT realiza as ações planejadas anteriormente e aprovadas pelo grupo gestor.

Etapa 12: Após a execução do plano, é realizada uma avaliação comparativa entre ações realizadas com previstas, ou seja, é realizado o controle dos planos de ação. Para este controle, os indicadores de desempenho estabelecidos devem ser avaliados criteriosamente. $\mathrm{O}$ controle do processo deve se dar de maneira contínua, retornando-se à Etapa 11 sempre que ocorra alguma discrepância entre o previsto e o realizado.

Etapa 13: Consiste no replanejamento das ações, uma vez que as mesmas podem não terem surtido o resultado esperado, ou ainda, não serem mais suficientes para a manutenção do processo.

\section{Caso: aplicação em uma indústria do setor metal-mecânico}

Esta seção tem por objetivo apresentar os principais aspectos e etapas envolvendo a implantação da MPEM em uma Indústria do setor metal-mecânico do estado do Rio Grande do Sul. Por decisão da empresa, um projeto piloto da MPEM foi implantado. A partir dos resultados obtidos com este piloto, os próprios profissionais da empresa ficaram responsáveis pelo restante da implantação. O caso é descrito dentro do cenário mais próximo da realidade, apresentando o conjunto de estratégias elaboradas para a alavancagem da gestão dos materiais na empresa em questão. Como forma de controle e monitoramento das estratégias para os materiais, optou-se por criar um conjunto de indicadores que proporcionassem um acompanhamento direto e pragmático da evolução dentro da empresa.

O projeto piloto foi realizado ao longo de cinco semanas. Nas três primeiras foram realizados dois encontros semanais, com duração de quatro horas cada. $\mathrm{Na}$ quarta semana foi elaborada a apresentação para a diretoria e na quinta semana o trabalho foi levado à diretoria, totalizando 8 encontros.

A seguir são descritas as atividades realizadas no caso, conforme as etapas do MGTP:

Etapa 1: O ponto de partida para a implementação da MPEM consistiu no alinhamento do corpo diretivo da empresa, relativamente à importância e potenciais resultados que podem ser atingidos com a adoção de novas práticas modernas de gestão de materiais na empresa. Primeiramente, foi estruturada uma apresentação abordando os elementos teóricos que sustentam a MPEM, os principais benefícios que a mesma potencialmente proporciona ao resultado econômico-financeiro e gerencial da empresa, e o método de implantação.

Etapa 2: Também realizada no primeiro encontro, consistiu em reunir todos os gerentes e supervisores para tratarem dos aspectos preliminares da implantação. A unidade fabril em que foi realizado o trabalho está dividida em minifábricas, e estas em linhas de produção. Cada minifábrica é administrada por um gerente distinto, e cada linha conta com um supervisor de produção. Além desses profissionais, também estiveram presentes, para participar do GT, representantes das áreas de qualidade, desenvolvimento de fornecedores, programação e controle da produção, logística, compras, custos e engenharia de produto. Ficou assim definida a equipe para desenvolver os trabalhos de implantação.

Etapa 3: No segundo encontro, com base nos produtos e materiais a serem tratados no projeto piloto de implantação da MPEM definidos, o GT definiu os produtos e materiais através de um processo de brainstorming. Como exemplo, foi selecionado o Produto Final A e os 
materiais: i) forjado; ii) graxeiras; iii) plásticos; e iv) extrudado.

Etapa 4: Passou-se então para o repasse conceitual do método, apresentando-se os pontos básicos, e assim as primeiras votações e cálculos foram realizados. O restante do segundo encontro foi destinado para o treinamento e repasse tecnológico.

Etapas 5 e 6: No terceiro encontro foram discutidos os critérios de classificação a serem utilizados, e foi atingido o consenso entre os mesmos. Em seguida, iniciou-se a elaboração preliminar da MPEM. Para tanto, o grupo de trabalho recebeu dois formulários:

a) um para a coleta de dados necessários para a construção do índice de influência nos resultados da empresa; e

b) um para a coleta dos dados para a formação do índice do risco de suprimento dos materiais.

De acordo com o método, cada participante recebeu um formulário deste tipo e o procedimento de votação foi repetido para todos os produtos selecionados. Apenas para a votação do índice de influência sobre os resultados foi necessário um encontro inteiro de quatro horas. Parte do quarto encontro foi reservada para a avaliação do formulário para análise do risco de suprimento do material em particular, não interessando a qual produto o mesmo estava associado.

Etapa 7 e Revisão da Etapa 6: Ao final do quarto encontro, iniciou-se a tabulação dos dados coletados, a fim de gerar as informações necessárias para a empresa.

Para auxiliar no tratamento dos dados coletados, foi utilizada uma planilha eletrônica pré-concebida, na qual todos os dados são informados e automaticamente é indicada a posição correspondente ao quadrante de cada material analisado. Após a inclusão dos respectivos dados de cada produto e material, o sistema gera um relatório resumindo os índices médios indicados pelos participantes do GT. As Tabelas 1 e 2 apresentam um exemplo da relação entre produto final $\mathrm{A}$ e o material forjado. Conforme a figura, o relatório apresenta a média ponderada em relação à influência nos resultados (para o caso do produto final A o índice é 3,73 ) e a média para o risco de suprimento (para o caso do forjado é 3,32). Ao mesmo tempo, a ferramenta constrói a MPEM graficamente, indicando o quadrante respectivo aos índices calculados (no caso da relação produto final A x forjado, 3,73 e 3,32), conforme mostra a Figura 5. Neste caso, o quadrante resultante na matriz é o correspondente aos componentes estratégicos. Este mesmo processo foi repetido para todas as relações entre produtos $\mathrm{X}$ materiais.

Os resultados finais da elaboração preliminar da MPEM para o Produto Final A foram os seguintes:

a) forjado $=$ componente estratégico;

b) extrudado = componente competitivo;
Tabela 1. Influência nos resultados. Índices do produto final $\mathrm{A} x$ forjado.

\begin{tabular}{lccc}
\hline & $\begin{array}{c}\text { Peso relativo } \\
\text { na competição }\end{array}$ & $\begin{array}{c}\text { Influência do } \\
\text { material }(\mathbf{0 , 5})\end{array}$ & $\begin{array}{c}\text { Influência } \\
\text { ponderada }\end{array}$ \\
\hline Custo & 0,21 & 3,10 & 0,65 \\
Qualidade & 0,36 & 4,40 & 1,58 \\
Tempo & 0,17 & 2,80 & 0,46 \\
Tecnologia & 0,27 & 3,90 & 1,03 \\
Total & 1,0 & & 3,73 \\
\hline
\end{tabular}

Tabela 2. Risco de suprimento. Índices do produto final A $x$ forjado.

\begin{tabular}{ll}
\hline Poder de barganha & 3,33 \\
Substituição & 3,42 \\
Rivalidade & 3,58 \\
Barreiras à entrada & 3,08 \\
Índice & 3,35 \\
\hline
\end{tabular}

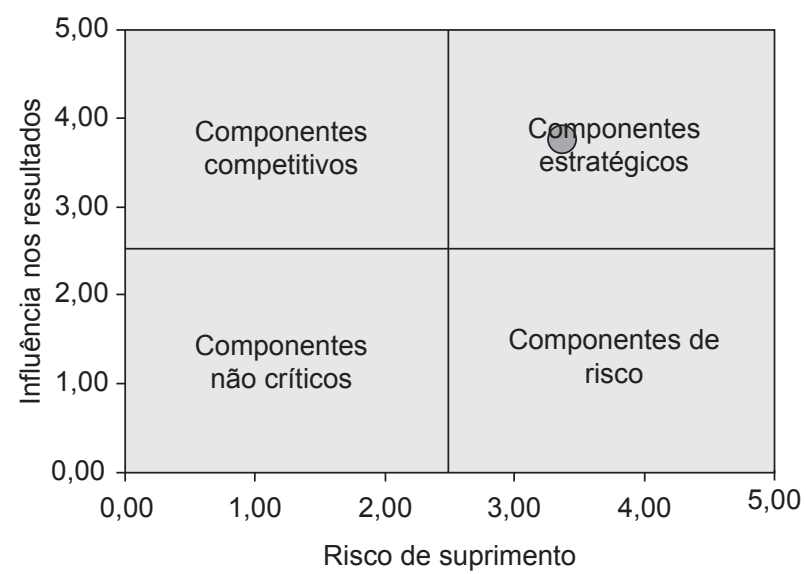

Figura 5. Posição na matriz - produto final A $x$ forjado. Índice de influência nos resultados: 3,73.

Índice de risco de suprimento: 3,35 .

c) graxeiras = componente não-crítico; $\mathrm{e}$

d) plásticos = componente de risco.

No quinto encontro, foram apresentados os resultados tabulados para o GT. O encontro foi conduzido de modo a estimular um amplo debate sobre os resultados atingidos. Cada caso foi analisado de modo a se obter consenso em relação ao posicionamento de cada material. Algumas divergências de pontos de vistas surgidas ao longo do encontro foram dirimidas ao final, porém outras requereram verificação e pequenas alterações. Neste caso, foi necessário gerar uma nova votação para os materiais reanalisados. Em relação à análise dos resultados obtidos no produto final A, observou-se uma discrepância relacionada ao material "plásticos". Na primeira votação/ tabulação, o posicionamento apontado foi componente de risco, indicando que apesar de não representar muito, em termos financeiros, para o produto em questão, este material poderia ser responsável pelo atraso na produção 
e entrega do produto. Porém, no caso do material "plásticos", de acordo com a maioria dos membros do GT, esta posição não representava a realidade. Esta situação foi gerada devido ao fato de existirem dois tipos específicos de plásticos na empresa. Os plásticos técnicos são componentes de risco, pois precisam ser tratados com cuidado, uma vez que os processos de fabricação são mais complexos. No caso dos plásticos comuns, estes são componentes não-críticos. Alguns componentes do GT não haviam percebido claramente a distinção e alguns estavam analisando os plásticos comuns, e outros os técnicos. sendo assim, optou-se por dividir o material "plásticos" em dois, existindo assim plásticos técnicos e plásticos comuns.

Etapa 8: Após completar a reanálise e as novas tabulações dos produtos e materiais que se fizeram necessários, o GT avançou para a Etapa 8, que consistiu na elaboração de propostas de gestão e ação para cada um dos segmentos da MPEM. Esta etapa foi desenvolvida no quinto e sexto encontro. A Tabela 3 apresenta algumas propostas sugeridas pelo GT.

A empresa ainda elaborou um conjunto de indicadores de desempenho para assegurar o controle estratégico dos materiais ao longo do tempo. O conjunto de indicadores selecionados foram os seguintes:

a) migração de itens: verificação periódica em termos de migração dos itens entre os quadrantes da matriz;

b) giro de estoque: acompanhar a evolução dos giros de estoque de materiais e registrar as melhorias decorridas dos planos de ação elaborados; e

c) fornecedores com qualidade assegurada: monitorar o número de fornecedores da empresa com qualidade de produto e fornecimento.
Etapa 9: Conforme o planejamento realizado no início da implantação piloto da MPEM, a semana seguinte ficou reservada para a elaboração da documentação e material que foi apresentado ao corpo diretivo da empresa.

Etapas 10, 11, 12 e 13: Encontram-se em realização na empresa, sendo geridas internamente, com base nos elementos conceituais e tecnológicos repassados durante a implantação da MPEM.

\section{Conclusões}

O presente artigo sugere, inicialmente, a necessidade das empresas industriais modernas proporem estratégias objetivas e eficazes para a gestão dos materiais. As formas tradicionais para abordar a questão (como a utilização apenas do método $\mathrm{ABC}$ ), não se mostram suficientes para esta tarefa, requerendo adaptações ou a adoção de abordagens alternativas.

Tradicionalmente, o método ABC leva em consideração somente os aspectos financeiros envolvidos na compra de materiais. Uma alternativa é o uso do método ABC com criticidade. No entanto, a forma preconizada de construção da classificação $\mathrm{ABC}$ com criticidade tende a apresentar uma hierarquia entre os elementos custo e criticidade. $\mathrm{O}$ reagrupamento de materiais por esse método pode conduzir a ações não completamente adequadas, do ponto de vista da gestão estratégica dos materiais. Por exemplo, torna-se difícil realizar uma avaliação crítica da migração de um item de uma categoria para outra.

Uma proposição prática para o equacionamento deste tipo de questão consiste em utilizar a MPEM como ferramenta estratégica para a gestão dos materiais, a qual pode ser agregada aos elementos de gestão vigentes. Este método permite segmentar os materiais em quatro quadrantes distintos: não-críticos, estratégicos, de risco e

Tabela 3. Propostas de ação geradas pelo GT.

\begin{tabular}{ll}
\hline \multicolumn{1}{c}{ Posição } & \multicolumn{1}{c}{ Propostas } \\
\hline $\begin{array}{l}\text { Componentes estratégicos } \\
\text { (forjado) }\end{array}$ & - conhecimento sobre o mercado potencial de fornecimento e redefinição do processo de seleção dos \\
& - fornecedores; \\
& - deftruturaçãão de um grupo de trabalho permanente entre empresa e fornecedor; \\
& - redução dos custos globais de transação e operação; e \\
& - intercâmbio de tecnologia entre empresa e fornecedores. \\
Componentes não-críticos & - redução do número de fornecedores; e \\
(graxeiras e plásticos comuns) & - estratégias de redução de custos: filiais de fornecedores próximos à empresa, leilão eletrônico, \\
& compras corporativas. \\
Componentes competitivos & - redução e qualificação da base de fornecedores; \\
(extrudado) & estudo do grupo estruturado para verificar as condições técnicas/econômicas da terceirização; \\
& - realização de kaizen com os fornecedores escolhidos; e \\
Componentes de risco & melhorias da qualidade e padronização dos componentes. \\
(plásticos técnicos) & reprojeto e padronização dos componentes; \\
& - celebração de contratos de longo prazo; e \\
\hline
\end{tabular}


competitivos. Para cada um dos quadrantes da MPEM o método prevê o desenvolvimento de estratégias diferenciadas de gestão dos materiais.

O uso da MPEM não impede o uso de classificações ABC. Uma possibilidade, por exemplo, é o uso de uma escala gráfica (cores ou diâmetros) associada ao volume de compras de cada item nos quadrantes da matriz (CAVANHA FILHO, 2001, 2003). Outro ponto de interface, em que a classificação $\mathrm{ABC}$ é um elemento muito importante, é o posicionamento dos materiais, no que tange à influência nos resultados. Nesse caso, a classificação ABC pode servir de subsídio para os processos de votação propostos no método da MPEM. No entanto, é necessário não confundir o método $\mathrm{ABC}$ com o resultado final obtido na Influência sobre os resultados da MPEM. Isto porque, além da dimensão custos (para a qual a construção e compreensão das curvas $\mathrm{ABC}$ é fundamental), a influência sobre os resultados também considera os critérios associados à qualidade, tempo e tecnologia.

O artigo propõe um método rigoroso e sistemático para operacionalizar a MPEM, levando em conta a realidade das empresas brasileiras, através da utilização do MGTP para a implantação da MPEM (ANTUNES; KLIPPEL, 2002). O artigo descreve a adaptação deste método em suas principais etapas, apresentando sua viabilidade através de um caso de implantação em uma empresa do setor metal-mecânico do Rio Grande do Sul.

A abordagem proposta permite uma maior clareza no que tange à real importância de cada material na formação da capacidade competitiva da empresa, dando prioridade aos elementos de maior impacto sobre a empresa, e assim possibilitando uma gestão mais eficaz dos materiais. Em particular, apresenta um instrumento de larga aplicabilidade, fácil compreensão e alta flexibilidade na consideração da dimensão estratégica da gestão de materiais. Este instru- mento é suportado por um método de implantação, que se destina a eliminar eventuais ambigüidades descritas nos processos tradicionais de classificação $\mathrm{ABC}$ e $\mathrm{ABC}$ com criticidade, no que tange à separação das classes. Além disso, a abordagem proposta explicita o impacto estratégico da alteração dos pontos de corte entre as classes, tanto na dimensão Influência sobre os resultados como na dimensão Risco de suprimento, permitindo ações mais focadas e evolutivas. Nesse sentido, a abordagem também abrange a elaboração de planos de ações explícitos para a gestão estratégica dos materiais, bem como seu acompanhamento através de indicadores, indo além das propostas preconizadas nos métodos de classificação, como o $\mathrm{ABC}$ ou o ABC com criticidade.

Em termos de limitações do presente artigo, pode-se relacionar:

a) trata-se de um estudo de caso e, portanto, não pode ser generalizado em suas conclusões (especialmente no que tange às sugestões de ação relacionadas às formas de gestão dos quadrantes da MPEM, uma vez que dependem de elementos estratégicos de cada empresa);

b) apresenta o processo de implantação da MPEM, mas não permite a avaliação de resultados práticos, os quais dependem da implantação da gestão da MPEM e da evolução dos indicadores de desempenho ao longo do tempo; e

c) é possível aprimorar o método através da realização de um maior número de estudos de casos.

Finalmente, a gestão eficaz dos materiais contribui para assegurar a alocação adequada de esforço de gestão sobre cada perfil de material, identificando e abordando, diferenciadamente, os de muita relevância para a empresa, tratados como pontos estratégicos para o negócio.

\title{
Strategic positioning matrix of materials: conceptualization, method and case study
}

\begin{abstract}
Materials management represents an important issue in a company's management since it can impact business global results. This paper presents the primary concepts which support and implement the strategic material positioning matrix. Additionally, a case in an automobile dealership is presented focusing on the necessary procedures to implement the matrix, as well as the necessity to effectively manage materials. Finally, the paper emphasizes the importance of material continuous management, involving the management segmentation at different quadrants of the Matrix and supported by a mechanism of performance measurement adopted by the company.
\end{abstract}

Keywords: Material management. Strategic management. Strategic positioning matrix. 
ANTUNES, J. A. V.; KLIPPEL, M. Matriz de posicionamento estratégico dos materiais: uma abordagem metodológica. In: XXII ENCONTRO NACIONAL DE ENGENHARIA DE PRODUÇÃO (ENEGEP), Curitiba, PR. Anais... Curitiba, 2002.

CARTER, R. C. Development of supply strategies. In: CAVINATO, J. L.; KAUFFMAN R. G. The purchasing handbook: a guide for the purchasing and supply professional. 6 ed. New York: McGraw-Hill, 1999. p. 81-98.

CAVANHA FILHO, A. O. Logística: Novos Modelos. 1. ed. São Paulo: Qualitymark, 2001.

CAVANHA FILHO, A. O. Canal fornecedor: a tecnologia internet no relacionamento com fornecedores. 1. ed. Rio de Janeiro: UNCTAD, 2003.

CAVINATO, J. L.; KAUFFMAN R. G. The purchasing handbook: a guide for the purchasing and supply professional. 6. ed. New York: McGraw-Hill, 1999.

DOBLER, D. W.; BURD, D. N. Purchasing and supply management: text and cases. 6. ed. New York: McGraw-Hill, 1996.
GRIECO, P. L. Supply management toolbox: how to manage your suppliers. 1. ed. West Palm Beach: PT Publications, Inc., 1995.

HARMON, R.L. Reinventando a Fábrica II. 1. ed. Rio de Janeiro: Campus, 1993.

HAVE, S. T. et al. Modelos de gestão: o que são e quando devem ser usados. 1. ed. São Paulo: Prentice Hall, 2003.

LEENDERS, M. R. et al. Purchasing and supply management. 12. ed. Boston: McGraw-Hill Irwin, 2001.

MARTINS, P. G.; ALT, P. R. C. Administração de materiais e recursos patrimoniais. 2. ed. São Paulo: Saraiva, 2004.

PORTER, M. E. Estratégia competitiva: técnicas para análise de indústrias e da concorrência. 15. ed. Rio de Janeiro: Campus, 1986.

SALVENDY, G. Handbook of industrial engineering. 2. ed. New York: John Wiley \& Sons, Inc, 1992.

\section{Sobre os autores}

\section{Marcelo Klippel}

KLIPPEL Consultores Associados e PRODUTTARE Consultores Associados, Rua Engenheiro Afonso Cavalcanti 54, Bela Vista, CEP 90440-110, Porto Alegre, RS, Brasil, e-mail: marcelo@klippel.com.br

\section{José Antonio Valle Antunes Júnior}

Departamento de Administração de Empresas, Universidade do Vale do Rio dos Sinos - UNISINOS e PRODUTTARE Consultores Associados,

Rua Engenheiro Afonso Cavalcanti 54, Bela Vista, CEP 90440-110, Porto Alegre, RS, Brasil,

e-mail: junico@ produttare.com.br

\section{Guilherme Luís Roehe Vaccaro}

Centro de Ciências Exatas e Tecnológicas, Programa de Pós-Graduação em Engenharia de Produção e Sistemas (PPGEPS), Universidade do Vale do Rio dos Sinos - UNISINOS e IMAGO Consultoria, Avenida Unisinos, 950 - Cristo Rei, CEP 93022-000, São Leopoldo, RS, Brasil e-mail: guilhermev@unisinos.br 